KIAS-P97003

DFTT 55/97

hep-ph/9709494

September 1997

\title{
When do neutrinos cease to oscillate?
}

\author{
C. Giunti ${ }^{\mathrm{a}}$, C. W. Kim ${ }^{\mathrm{b}, c}$, and U. W. Lee ${ }^{\mathrm{d}}$ \\ a INFN, Sez. di Torino, and Dip. di Fisica Teorica, Univ. di Torino, I-10125 Torino, Italy \\ b Department of Physics \& Astronomy, The Johns Hopkins University, Baltimore, MD 21218 \\ c School of Physics, Korea Institute for Advance Study, Seoul 130-012, Republic of Korea \\ d Department of Physics, Mokpo National University, Chonnam 534-729, Republic of Korea
}

(September 29, 1997)

\begin{abstract}
In order to investigate when neutrinos cease to oscillate in the framework of quantum field theory, we have reexamined the wave packet treatment of neutrino oscillations by taking different sizes of the wave packets of the particles involved in the production and detection processes. The treatment is shown to be considerably simplified by using the Grimus-Stockinger theorem which enables us to carry out the integration over the momentum of the propagating neutrino. Our new results confirm the recent observation by Kiers, Nussinov and Weiss that a precise measurement of the energies of the particles involved in the detection process would increase the coherence length. We also present a precise definition of the coherence length beyond which neutrinos cease to oscillate.
\end{abstract}

PACS number(s): 13.15.+g, 14.60.Pq 
A rigorous treatment of neutrino oscillations requires the study of the processes in which neutrinos are produced and detected [1 9]. Following the first attempts [1] to develop a proper use of the wave packet formalism, we have carried out detailed calculations in both quantum mechanics [4] and quantum field theory [5], with a quantitative derivation of the coherence length for neutrino oscillations. Due to the complexity of calculations, however, in [5] it was assumed that the sizes of the wave packets of the initial and final particles are all the same.

Recently, Grimus and Stockinger [8] proved an elegant and very useful theorem that allows to simplify the wave packet treatment of neutrino oscillations. Taking advantage of this theorem, we have re-derived the neutrino oscillation formula in the general case in which all the particles involved in the production and detection processes have different wave packet sizes. Our new result confirms in the framework of a quantum field theoretical approach an interesting observation presented in [7] that an accurate measurement of the energies of the particles involved in the detection process leads to an increase of the coherence length for neutrino oscillations.

Let us consider the flavor-changing process

$$
\begin{aligned}
P_{I} \rightarrow P_{F}+\ell_{\alpha}^{+}+ & \nu_{\alpha} \\
& \downarrow\left(\nu_{\alpha} \rightarrow \nu_{\beta}\right) \\
& \nu_{\beta}+D_{I} \rightarrow D_{F}+\ell_{\beta}^{-},
\end{aligned}
$$

where $P_{I}$ and $P_{F}\left(D_{I}\right.$ and $\left.D_{F}\right)$ are the initial and final production (detection) particles. The process (11) takes place through the intermediate propagation of a neutrino, which oscillates from flavor $\alpha$ to flavor $\beta$ (here $\alpha, \beta=e, \mu, \tau)$. In the process (1), the production and detection interactions are localized at the coordinates $\left(\vec{X}_{P}, T_{P}\right)$ and $\left(\vec{X}_{D}, T_{D}\right)$, respectively.

The form of the wave functions of the initial and final particles involved in the process (11) is determined by how the initial particles are prepared and how the final particles are detected. In the following, we will assume, for simplicity, Gaussian wave functions, whose wave packet forms in momentum and coordinate space are given in [5]. The wave packets in momentum space are assumed to be sharply peaked around their average momenta, which are denoted by $\left\langle\vec{p}_{k}\right\rangle$, where $k=P_{I}, P_{F}, \alpha, D_{I}, D_{F}, \beta$. The corresponding average energies $\left\langle E_{k}\right\rangle$ are given by $\left\langle E_{k}\right\rangle=\sqrt{\left\langle\vec{p}_{k}\right\rangle^{2}+m_{k}^{2}}$, where $m_{k}$ is the mass of the $k^{\text {th }}$ particle. In order to make a realistic calculation, we will consider a different spatial width $\sigma_{x k}$ for the wave packet of each particle involved in the process (1). Let us emphasize that the localization of the particles does not require necessarily the action of a man-made apparatus, but can be determined by the environment in which the process (1) takes place.

Following the method presented in [5], the amplitude of the process (11) can be written as (see Eq.(8) of [5])

$$
\begin{aligned}
& A_{\alpha \beta}(\vec{L}, T) \propto \sum_{a} \mathcal{U}_{\alpha a}^{*} \mathcal{U}_{\beta a} \int \frac{\mathrm{d}^{4} q}{(2 \pi)^{4}} \bar{U}_{D} \frac{\not d}{q^{2}-m_{a}^{2}+i \epsilon} V_{P} \exp \left[-i q_{0} T+i \vec{q} \cdot \vec{L}\right] \\
& \quad \times \int \mathrm{d}^{4} x_{1} \exp \left[-i\left(E_{P}-q_{0}\right) t_{1}+i\left(\vec{p}_{P}-\vec{q}\right) \cdot \vec{x}_{1}-\frac{\vec{x}_{1}^{2}-2 \vec{v}_{P} \cdot \vec{x}_{1} t_{1}+\Sigma_{P} t_{1}^{2}}{4 \sigma_{x P}^{2}}\right] \\
& \quad \times \int \mathrm{d}^{4} x_{2} \exp \left[-i\left(E_{D}+q_{0}\right) t_{2}+i\left(\vec{p}_{D}+\vec{q}\right) \cdot \vec{x}_{2}-\frac{\vec{x}_{2}^{2}-2 \vec{v}_{D} \cdot \vec{x}_{2} t_{2}+\Sigma_{D} t_{2}^{2}}{4 \sigma_{x D}^{2}}\right],
\end{aligned}
$$


where the index $a$ denotes the neutrino field $\nu_{a}$ with mass $m_{a}, \mathcal{U}$ is the neutrino mixing matrix, $\vec{L} \equiv \vec{X}_{D}-\vec{X}_{P}$ is the macroscopic distance vector from the neutrino production point to the neutrino detection point and $T \equiv T_{D}-T_{P}$ is the macroscopic time interval $T$ between neutrino production and detection. In Eq.(2) we have defined various quantities relative to the production process as follows (the corresponding quantities relative to the detection process are denoted with $P$ replaced by $D$ and $\alpha$ replaced by $\beta$ in the subscript). $E_{P}$ and $\vec{p}_{P}$ are given by

$$
\begin{aligned}
& E_{P} \equiv\left\langle E_{P_{I}}\right\rangle-\left\langle E_{P_{F}}\right\rangle-\left\langle E_{\alpha}\right\rangle, \\
& \vec{p}_{P} \equiv\left\langle\vec{p}_{P_{I}}\right\rangle-\left\langle\vec{p}_{P_{F}}\right\rangle-\left\langle\vec{p}_{\alpha}\right\rangle .
\end{aligned}
$$

The quantity $\sigma_{x P}$, given by

$$
\frac{1}{\sigma_{x P}^{2}} \equiv \frac{1}{\sigma_{x P_{I}}^{2}}+\frac{1}{\sigma_{x P_{F}}^{2}}+\frac{1}{\sigma_{x \alpha}^{2}},
$$

can be considered as the effective size of the production process. Notice that the width of the wave packet of the most localized particle (i.e. the one with the smallest $\sigma_{x}$ ) dominates in the determination of $\sigma_{x P}$. For example, in [7] it is argued that the width $\sigma_{x e}$ of the captured electron in the production process of ${ }^{7} \mathrm{Be}$ solar neutrinos, $e^{-}+{ }^{7} \mathrm{Be} \rightarrow{ }^{7} \mathrm{Li}+\nu_{e}$, is much smaller than the widths of the wave packets of the other particles involved in the process. Therefore, in this case $\sigma_{x P} \simeq \sigma_{x e}$ (the formalism presented here can be obviously generalized for the case of more than one initial particle).

In a quantum-mechanical framework (see [1, [4,7]) the size of the production process determines the size of the wave packet of the propagating neutrino. On the other hand, in the approach adopted here the neutrino is treated as a "virtual" particle propagating between the production and detection vertices, whose properties are defined by the production and detection interactions in an equal way. Hence, any attempt to define a neutrino wave packet in the framework of the calculation presented here would lead to a definition of its size in equal terms of both $\sigma_{x P}$ and $\sigma_{x D}$. Such an interpretation is unacceptable from a causal point of view. However, the present approach does not need an interpretation in terms of neutrino properties, because the neutrino is not directly observed, and we will show that, nevertheless, it leads to a rigorous derivation of the oscillation probability, including the effect of the coherence length.

The quantities $\vec{v}_{P}$ and $\Sigma_{P}$ in Eq.(2) are given by

$$
\begin{aligned}
& \vec{v}_{P} \equiv \sigma_{x P}^{2}\left(\frac{\vec{v}_{P_{I}}}{\sigma_{x P_{I}}^{2}}+\frac{\vec{v}_{P_{F}}}{\sigma_{x P_{F}}^{2}}+\frac{\vec{v}_{\alpha}}{\sigma_{x \alpha}^{2}}\right), \\
& \Sigma_{P} \equiv \sigma_{x P}^{2}\left(\frac{\vec{v}_{P_{I}}^{2}}{\sigma_{x P_{I}}^{2}}+\frac{\vec{v}_{P_{F}}^{2}}{\sigma_{x P_{F}}^{2}}+\frac{\vec{v}_{\alpha}^{2}}{\sigma_{x \alpha}^{2}}\right),
\end{aligned}
$$

where $\vec{v}_{k} \equiv\left\langle\vec{p}_{k}\right\rangle /\left\langle E_{k}\right\rangle$ is the group velocity of the $k^{\text {th }}$ particle. Finally, the definitions of $\bar{U}_{D}$ and $V_{P}$ are given by

$$
\begin{aligned}
& \bar{U}_{D} \equiv J_{\rho}^{D}\left(\left\langle\vec{p}_{D_{F}}\right\rangle,\left\langle\vec{p}_{D_{I}}\right\rangle\right) \bar{u}_{\beta}\left(\left\langle\vec{p}_{\beta}\right\rangle\right) \gamma^{\rho}\left(1+\gamma_{5}\right), \\
& V_{P} \equiv\left(1+\gamma_{5}\right) \gamma^{\rho} v_{\alpha}\left(\left\langle\vec{p}_{\alpha}\right\rangle\right) J_{\rho}^{P}\left(\left\langle\vec{p}_{P_{F}}\right\rangle,\left\langle\vec{p}_{P_{I}}\right\rangle\right),
\end{aligned}
$$


where $J_{\rho}^{P}\left(\left\langle\vec{p}_{P_{F}}\right\rangle,\left\langle\vec{p}_{P_{I}}\right\rangle\right)$ and $J_{\rho}^{D}\left(\left\langle\vec{p}_{D_{F}}\right\rangle,\left\langle\vec{p}_{D_{I}}\right\rangle\right)$ are the matrix elements of the weak currents of the production and detection particles.

Carrying out the gaussian integrals over $x_{1}$ and $x_{2}$, we obtain

$$
\begin{aligned}
A_{\alpha \beta}(\vec{L}, T) \propto \sum_{a} \mathcal{U}_{\alpha a}^{*} \mathcal{U}_{\beta a} \int \frac{\mathrm{d}^{4} q}{(2 \pi)^{4}} \bar{U}_{D} \frac{\not d}{q^{2}-m_{a}^{2}+i \epsilon} V_{P} \exp \left[-i q_{0} T+i \vec{q} \cdot \vec{L}\right] \\
\times \exp \left[-\frac{\left(\vec{p}_{P}-\vec{q}\right)^{2}}{4 \sigma_{p P}^{2}}-\frac{\left[\left(E_{P}-q_{0}\right)-\left(\vec{p}_{P}-\vec{q}\right) \cdot \vec{v}_{P}\right]^{2}}{4 \sigma_{p P}^{2} \lambda_{P}}\right. \\
\left.-\frac{\left(\vec{p}_{D}+\vec{q}\right)^{2}}{4 \sigma_{p D}^{2}}-\frac{\left[\left(E_{D}+q_{0}\right)-\left(\vec{p}_{D}+\vec{q}\right) \cdot \vec{v}_{D}\right]^{2}}{4 \sigma_{p D}^{2} \lambda_{D}}\right],
\end{aligned}
$$

where we have defined $\lambda_{P(D)} \equiv \Sigma_{P(D)}-\vec{v}_{P(D)}^{2}$ and $\sigma_{p P(D)}$ is defined by the relation $\sigma_{x P(D)} \sigma_{p P(D)}=1 / 2$. Notice that the integrations over $x_{1}$ and $x_{2}$ in Eq.(2) did not produce the usual $\delta$-functions representing energy-momentum conservation at each interaction vertex. Indeed, in the wave packet treatment the energies and momenta of the particles involved in the process under consideration do not have a precise value, allowing for the uncertainty in energy-momentum conservation that is necessary for the occurrence of neutrino oscillations (see [3]).

From Eqs.(6) and (7) one can see that $0 \leq \lambda_{P(D)}<1$. Hence, it is natural to ask what happens if $\lambda_{P}=0$ and/or $\lambda_{D}=0$. Let us consider, for simplicity, the case of only $\lambda_{P}=0$. This situation could arise, for example, if only the initial particle $P_{I}$ in the production process is observed with a good localization at $\vec{X}_{P}$ and is described by a wave packet, whereas all the particles in the final state are described by plane waves. In this case we have $\sigma_{x P}=\sigma_{x P_{I}}, \vec{v}_{P}=\vec{v}_{P_{I}}$ and $\Sigma_{P}=\vec{v}_{P_{I}}^{2}$, leading to $\lambda_{D}=0$. From Eq.(2) one can see that $\Sigma_{P}=\vec{v}_{P_{I}}^{2}$ implies exact energy conservation in the production process $\left(\delta\left(q_{0}-E_{P}\right)\right)$. If $\vec{v}_{P} \neq 0$, there is also exact momentum conservation in the production process $\left(\delta^{3}\left(\vec{q}-\vec{p}_{P}\right)\right)$ and neutrino oscillations do not occur because exact energy-momentum conservation in the production process can be satisfied only by one of the neutrino mass eigenstates, excluding the coherent production of more than one neutrino mass eigenstates that is necessary for neutrino oscillations. This fact is also clear in coordinate space: if $\vec{v}_{P} \neq 0$ only the trajectory of $P_{I}$ is known, which is not sufficient to determine the position $\vec{X}_{P}$ and the time $T_{P}$ of the production process. Hence, the distance $\vec{L}$ and the time interval $T$ are not defined and oscillations are not observable. On the other hand, in the special case $\vec{v}_{P}=0$ there is no exact momentum conservation in the production process (although energy is exactly conserved) and neutrino oscillations are observable. Physically this situation corresponds to have the initial particle $P_{I}$ at rest, in such a way that $\vec{X}_{P}$ is known and the distance $\vec{L}$ is defined, although $T_{P}$ and $T$ are not known (similar scenarios are discussed in [6,8]). However, as stated above, the calculation presented here is based on the assumption that all the initial and final particles in the process (11) are observed and their wave packets are sharply peaked around the corresponding average momentum. Therefore, the case $\lambda_{P}=0$ (and/or $\lambda_{D}=0$ ) corresponds to a different physical process from the one under consideration, which can be discussed modifying the calculation presented here in an appropriate way. Under our assumptions, $\lambda_{P}$ and $\lambda_{D}$ are dimensionless quantities of order $10^{-1}$. For example, if 
the production process is pion decay at rest, we have $\lambda_{P}=\frac{\sigma_{x P}^{2}}{\sigma_{x \alpha}^{2}}\left(1-\frac{\sigma_{x P}^{2}}{\sigma_{x \alpha}^{2}}\right) \vec{v}_{\alpha}^{2}$ with $\vec{v}_{\alpha}^{2}=$ $\left(m_{\pi}^{2}-m_{\alpha}^{2}\right)^{2} /\left(m_{\pi}^{2}+m_{\alpha}^{2}\right)^{2}$, i.e. $\vec{v}_{\alpha}^{2} \simeq 7 \times 10^{-2}$ for $\alpha=\mu$ and $\vec{v}_{\alpha}^{2} \simeq 1$ for $\alpha=e$.

Resuming the calculation of the amplitude (10), we perform the integral over $\vec{q}$ using the Grimus-Stockinger theorem [8]:

$$
\int \mathrm{d}^{3} q \frac{\phi(\vec{q}) \mathrm{e}^{i \vec{q} \cdot \vec{L}}}{q_{a}^{2}-\vec{q}^{2}+i \epsilon} \stackrel{L \rightarrow \infty}{\longrightarrow}-\frac{2 \pi^{2}}{L} \phi\left(q_{a} \vec{\ell}\right) \mathrm{e}^{i q_{a} L},
$$

with $L \equiv|\vec{L}|, \vec{\ell} \equiv \vec{L} / L$ and $q_{a}=\sqrt{q^{0^{2}}-m_{a}^{2}}$. This theorem is valid for a function $\phi$ which is differentiable at least three times such that $\phi$ itself and its first and second derivatives decrease at least as $1 / \vec{q}^{2}$ as $|\vec{q}| \rightarrow \infty$. This is precisely our case. The remaining integration over $q^{0}$ can be done with a saddle-point approximation at $q^{0}=E_{a}$, with $E_{a}$ given by the relation

$$
\begin{gathered}
E_{a} \frac{p_{a}-\vec{\ell} \cdot \vec{p}_{P}}{\sigma_{p P}^{2}}-\frac{\left(p_{a}-E_{a} \vec{\ell} \cdot \vec{v}_{P}\right)\left[\left(E_{P}-E_{a}\right)-\left(\vec{p}_{P}-p_{a} \vec{\ell}\right) \cdot \vec{v}_{P}\right]}{\sigma_{p P}^{2} \lambda_{P}} \\
+E_{a} \frac{p_{a}+\vec{\ell} \cdot \vec{p}_{D}}{\sigma_{p D}^{2}}+\frac{\left(p_{a}-E_{a} \vec{\ell} \cdot \vec{v}_{D}\right)\left[\left(E_{D}+E_{a}\right)-\left(\vec{p}_{D}+p_{a} \vec{\ell}\right) \cdot \vec{v}_{D}\right]}{\sigma_{p D}^{2} \lambda_{D}}=0,
\end{gathered}
$$

where $p_{a} \equiv q_{a}\left(E_{a}\right)=\sqrt{E_{a}^{2}-m_{a}^{2}}$. The quantities $E_{a}$ and $p_{a}$ can be interpreted as the effective energy and momentum of the $a^{\text {th }}$ neutrino mass eigenstate propagating between the production and detection vertices. In order to simplify our discussion, in the following we omit explicit terms of the order $m_{a}^{2} / E^{2}$, which are negligible for relativistic neutrinos. Thus, for $L \rightarrow \infty$ we have

$$
A_{\alpha \beta}(\vec{L}, T) \propto \frac{1}{L} \sum_{a} \mathcal{U}_{\alpha a}^{*} \mathcal{U}_{\beta a} \mathcal{A}_{a} \frac{1}{\sqrt{\Omega_{a}}} \exp \left[-i E_{a} T+i p_{a} L-S_{a}\left(E_{a}\right)-\frac{1}{2} \frac{\left(L-v_{a} T\right)^{2}}{v_{a}^{2} \Omega_{a}}\right]
$$

where $v_{a} \equiv p_{a} / E_{a}$,

$$
\begin{aligned}
\mathcal{A}_{a} \equiv & \bar{U}_{D}\left(E_{a} \gamma^{0}-p_{a} \vec{\ell} \cdot \vec{\gamma}\right) V_{P} \\
\Omega_{a} \equiv & \frac{1}{2 v_{a}^{2}}\left\{\frac{1}{\sigma_{p P}^{2}}+\frac{1}{\sigma_{p D}^{2}}+\frac{\left(v_{a}-\vec{\ell} \cdot \vec{v}_{P}\right)^{2}}{\sigma_{p P}^{2} \lambda_{P}}+\frac{\left(v_{a}-\vec{\ell} \cdot \vec{v}_{D}\right)^{2}}{\sigma_{p D}^{2} \lambda_{D}}\right\}+O\left(\frac{m_{a}^{2}}{E_{a}^{2}}\right), \\
S_{a}\left(E_{a} \equiv \equiv\right. & \frac{\left(\vec{p}_{P}-p_{a} \vec{\ell}\right)^{2}}{4 \sigma_{p P}^{2}}+\frac{\left[\left(E_{P}-E_{a}\right)-\left(\vec{p}_{P}-p_{a} \vec{\ell}\right) \cdot \vec{v}_{P}\right]^{2}}{4 \sigma_{p P}^{2} \lambda_{P}} \\
& +\frac{\left(\vec{p}_{D}+p_{a} \vec{\ell}\right)^{2}}{4 \sigma_{p D}^{2}}+\frac{\left[\left(E_{D}+E_{a}\right)-\left(\vec{p}_{D}+p_{a} \vec{\ell}\right) \cdot \vec{v}_{D}\right]^{2}}{4 \sigma_{p D}^{2} \lambda_{D}} .
\end{aligned}
$$

The probability of the process (四) is proportional to $\left|A_{\alpha \beta}(\vec{L}, T)\right|^{2}$, but in a practical experimental setting $\vec{L}$ is usually a fixed and known quantity, whereas $T$ is not measured. 
Therefore, the oscillation probability at a given distance $\vec{L}$ is given by the time average of $\left|A_{\alpha \beta}(\vec{L}, T)\right|^{2}$, which leads to

$$
\begin{aligned}
P_{\alpha \beta}(\vec{L}) \propto \frac{1}{L^{2}} \sum_{a, b} & \mathcal{A}_{a} \mathcal{A}_{b}^{*} \mathcal{U}_{\alpha a}^{*} \mathcal{U}_{\beta a} \mathcal{U}_{\alpha b} \mathcal{U}_{\beta b}^{*} \frac{1}{\sqrt{\Omega_{a}+\Omega_{b}}} \exp \left[-S_{a}\left(E_{a}\right)-S_{b}\left(E_{b}\right)\right] \\
\times \exp & {\left[-i\left(\left(E_{a}-E_{b}\right) \frac{v_{a} \Omega_{a}+v_{b} \Omega_{b}}{v_{a} v_{b}\left(\Omega_{a}+\Omega_{b}\right)}-\left(p_{a}-p_{b}\right)\right) L\right] } \\
\times \exp & {\left[-\frac{L^{2}}{2} \frac{\left(v_{a}-v_{b}\right)^{2}}{v_{a}^{2} v_{b}^{2}\left(\Omega_{a}+\Omega_{b}\right)}-\frac{1}{2}\left(E_{a}-E_{b}\right)^{2} \frac{\Omega_{a} \Omega_{b}}{\Omega_{a}+\Omega_{b}}\right] }
\end{aligned}
$$

The overall factor $1 / L^{2}$ represents the geometrical decrease of the neutrino flux. Notice that the probability (17) depends not only on the modulus $L$ of the distance, but also on its direction $\vec{\ell}$, which is contained in $\mathcal{A}_{a}, \Omega_{a}$ and $S_{a}\left(E_{a}\right)$ (see Eqs.(14)-(16)) and determines $E_{a}$ through Eq.(12). This is due to the fact that the wave packets of the external particles are assumed to be known and the term $\exp \left[-S_{a}\left(E_{a}\right)-S_{b}\left(E_{b}\right)\right]$ in Eq.(17) guarantees that the probability of the process (1) is not negligible only when both $\vec{p}_{P}$ and $\vec{p}_{D}$ are aligned with $\vec{\ell}$ within the uncertainty allowed by the sizes of the wave packets.

Since we are concerned with relativistic neutrinos, we approximate

$$
E_{a} \simeq E+\rho \frac{m_{a}^{2}}{2 E}
$$

with $E$ and $\rho$ determined, respectively, by the expansion of Eq.(12) at zeroth and first order in powers of $m_{a}^{2} / E^{2}$. Equation (18) leads to the approximations $p_{a} \simeq E+(\rho-1) m_{a}^{2} / 2 E$, $v_{a} \simeq 1-m_{a}^{2} / 2 E^{2}$ and $\Omega_{a} \simeq 2 \omega \sigma_{x}^{2}$, with

$$
\begin{aligned}
& \sigma_{x}^{2} \equiv \sigma_{x P}^{2}+\sigma_{x D}^{2}, \\
& \omega \equiv 1+\frac{\sigma_{x P}^{2}}{\sigma_{x}^{2}} \frac{\left(1-\vec{\ell} \cdot \vec{v}_{P}\right)^{2}}{\lambda_{P}}+\frac{\sigma_{x D}^{2}}{\sigma_{x}^{2}} \frac{\left(1-\vec{\ell} \cdot \vec{v}_{D}\right)^{2}}{\lambda_{D}} .
\end{aligned}
$$

From Eq.(16) one can see that in the relativistic approximation $S_{a}\left(E_{a}\right)$ is minimum for $\vec{p}_{P} \simeq-\vec{p}_{D} \simeq E \vec{\ell}$ and $E_{P} \simeq-E_{D} \simeq E$. These are the values of the kinematical parameters for which the probability (17) of the process (1) is not negligibly small (without considering the geometrical suppression factor $\left.1 / L^{2}\right)$. Hence, energy-momentum conservation is approximately satisfied in order to guarantee the observability of the process (11) and this approximate conservation determines the value of the effective neutrino energy $E$. From Eq.(16) one can also see that $\rho$ is a dimensionless quantity of order unity. On the other hand, $\omega$ could be rather large if $\lambda_{P(D)}$ is small. For example, if the production process is pion decay at rest with $\alpha=\mu, \lambda_{D} \sim 1$ and $\sigma_{x} \sim \sigma_{x P}$, we have $\omega \sim 10$.

In the relativistic approximation one can factorize out of the sum over the mass eigenstate indices $a$ and $b$ in Eq.(17) all the quantities that do not vanish in the zeroth order of the expansion in powers of $m_{a}^{2} / E^{2}\left(\right.$ e.g. $\mathcal{A}_{a}$ and $S_{a}\left(E_{a}\right)$ ). Therefore, in the relativistic approximation for the flavor-changing probability we have 


$$
P_{\alpha \beta}(\vec{L})=\sum_{a, b} \mathcal{U}_{\alpha a}^{*} \mathcal{U}_{\beta a} \mathcal{U}_{\alpha b} \mathcal{U}_{\beta b}^{*} \exp \left[-2 \pi i \frac{L}{L_{a b}^{\mathrm{osc}}}-\left(\frac{L}{L_{a b}^{\mathrm{coh}}}\right)^{2}-2 \pi^{2} \omega \rho^{2}\left(\frac{\sigma_{x}}{L_{a b}^{\mathrm{osc}}}\right)^{2}\right]
$$

with the oscillation lengths $L_{a b}^{\mathrm{osc}}$ and the coherence lengths $L_{a b}^{\text {coh }}$, for $a \neq b$, given by

$$
\begin{aligned}
L_{a b}^{\mathrm{osc}} & \equiv \frac{4 \pi E}{\Delta m_{a b}^{2}} \\
L_{a b}^{\mathrm{coh}} & \equiv 2 \sqrt{2 \omega} \frac{2 E^{2}}{\left|\Delta m_{a b}^{2}\right|} \sigma_{x},
\end{aligned}
$$

where $\Delta m_{a b}^{2} \equiv m_{a}^{2}-m_{b}^{2}$.

The transition probability (21) has the same form as that given in Eq.(23) of [5], which was obtained in the framework of quantum field theory with wave packets. However, the coefficients that appear in the expression of the coherence length and in front of the third term in the exponential in Eq.(21) are different from those of [5]. There are two reasons for these differences: 1) in 5] we assumed the same spatial width for all the wave packets of the external particles involved in the process (1), whereas here these widths can be different; 2) in (5) we integrated over the momenta of the final particles, whereas here we assume these momenta to be measured.

The first term in the exponential in Eq.(21) is the usual oscillating phase which gives rise to neutrino oscillations. The second term causes a quadratical decrease of $P_{\alpha \beta}(\vec{L})$ with the distance $L$ and determines how far the oscillations take place. For $L \gtrsim L_{a b}^{\text {coh }}$ this term suppresses the interference of the neutrino mass eigenstates $\nu_{a}$ and $\nu_{b}$, leading to the disappearance of the oscillations due to $L_{a b}^{\text {osc }}$ for $L \gg L_{a b}^{\text {coh }}$. If $L \gg L_{a b}^{\text {coh }}$ for all $a \neq b$, all the oscillating terms in the probability (21) are suppressed, leading to a constant transition probability $P_{\alpha \beta}=\sum_{a}\left|\mathcal{U}_{\alpha a}\right|^{2}\left|\mathcal{U}_{\beta a}\right|^{2}$. The third term in the exponential in Eq.(21), which is due to the time integration, i.e. to the lack of time measurements, implies that the interference terms are also washed out if $\sigma_{x}$ is larger than the neutrino oscillation length.

Apart from the factor $2 \sqrt{2 \omega}$, the coherence length given in Eq.(23) is similar to that obtained by physical intuition in [1-3] and in a quantum mechanical wave-packet treatment in [4]. But there is a very important difference between our result and the previous formulas for the coherence length. The coherence length is usually defined as the distance at which the separation between the wave packets of different massive neutrinos propagating with group velocities $v_{a} \simeq 1-m_{a}^{2} / E^{2}$ is equal to $\sigma_{x}$, i.e.

$$
L_{a b}^{\mathrm{coh}} \equiv \frac{\sigma_{x}}{\left|v_{a}-v_{b}\right|}=\frac{2 E^{2}}{\left|\Delta m_{a b}^{2}\right|} \sigma_{x},
$$

where $\sigma_{x}$ is the width of the propagating neutrino wave packet, which depends on the neutrino production mechanism. However, our calculations show that the proper definition of the $\sigma_{x}$ that determines the coherence length must also include information on the neutrino detection mechanism. We presented a rigorous definition of $\sigma_{x}$ in Eq.(19). Defining $\sigma_{p}$ with the relation $\sigma_{x} \sigma_{p}=1 / 2$, we have

$$
\frac{1}{\sigma_{p}^{2}} \equiv \frac{1}{\sigma_{p P}^{2}}+\frac{1}{\sigma_{p D}^{2}}
$$


Hence, it is clear that the smaller of $\sigma_{p P}$ and $\sigma_{p D}$ dominates the value of $\sigma_{p}$. If $\sigma_{p D}$ is much smaller than $\sigma_{p P}$, then $\sigma_{p} \simeq \sigma_{p D}$ and $\sigma_{x} \simeq \sigma_{x D}$. Therefore, a precise measurement of the momentum of all the particles involved in the neutrino detection process[ implies a very small $\sigma_{p} \simeq \sigma_{p D}$, leading to a very large $\sigma_{x} \simeq \sigma_{x D}$ and a very large coherence length. Thus, our formulation with wave packets confirms the observation in [7] and provides a simple method to estimate the $\sigma_{x}$ that determines the coherence length through Eq.(23): $\sigma_{x} \simeq$ $\sqrt{\sigma_{x P}^{2}+\sigma_{x D}^{2}}$, where $\sigma_{x P}$ and $\sigma_{x D}$ are the estimated sizes of the production and detection processes, respectively.

The presence of the factor $2 \sqrt{2 \omega}$ in the expression (23) of the coherence length can imply a non-negligible increase of $L_{a b}^{\mathrm{coh}}$ with respect to the usual definition (24). For example, as we have already mentioned, if the production process is pion decay at rest with $\alpha=\mu$ and $\lambda_{D} \sim 1$, we have $\omega \sim 10$, which gives $2 \sqrt{2 \omega} \sim 10$. Since $\omega$ is large if $\lambda_{P(D)}$ is small, i.e. if all the particles involved in the production (detection) process have a very low velocity, this increase of the coherence length is due to the fact that the overlap of the wave packets of slow particles last longer and there is more time available for the production (detection) process to emit (absorb) coherently the intermediate superposition of massive neutrinos. Indeed, one can estimate, for example, that the time $\Delta t_{P}$ available for the production process is of the order of $\sigma_{x P} /\left|\vec{v}_{P}\right|$ and, if $\left|\vec{v}_{P}\right|$ is small, $\lambda_{D} \sim 1$ and $\sigma_{x} \sim \sigma_{x P}$, we have $\omega \sim 1 /\left|\vec{v}_{P}\right|^{2} \sim\left(\Delta t_{P} / \sigma_{x}\right)^{2}$. Hence, one can see that a small $\left|\vec{v}_{P}\right|$ implies a large $\Delta t_{P}$, a large $\omega$ and a large coherence length.

On the other hand, it must be noticed that a large value of $\omega$ increases the contribution of the third term in the exponential in the transition probability (21) and could enhance its suppression of the interference terms if $\sigma_{x} \sim L_{a b}^{\text {osc }}$. This is due to the fact that when the time available for the production (detection) process is longer than $L_{a b}^{\text {osc }}$, the interference of the neutrino mass eigenstates $\nu_{a}$ and $\nu_{b}$ is washed out. For example, if $\omega \sim\left(\Delta t_{P} / \sigma_{x}\right)^{2}$ as in the example above, we have $\omega\left(\sigma_{x} / L_{a b}^{\text {osc }}\right)^{2} \sim\left(\Delta t_{P} / L_{a b}^{\text {osc }}\right)^{2}$ and the corresponding oscillating term in (21) is suppressed if $\Delta t_{P} \gg L_{a b}^{\text {osc }}$.

In summary, the Grimus-Stockinger theorem considerably simplifies the wave packet treatment. The neutrino oscillations due to $L_{a b}^{\text {osc }}$ disappear when the distance $L$ between neutrino production and detection is much larger than the coherence length $L_{a b}^{\text {coh }}$ given by Eq.(23) with Eq.(19), which is the most complete expression derived so far. The authors of [7] pointed out that one can observe neutrino oscillations beyond the usually defined coherence distance by a precise measurement of neutrino energy. Our calculation confirms their claim in the framework of a quantum field theoretical calculation. Another result of our calculation is that, because of the lack of time measurements, the neutrino oscillations due to $L_{a b}^{\text {osc }}$ can be observed only if $\sigma_{x}$ is smaller than $L_{a b}^{\text {osc }}$. Otherwise, the interference of the mass eigenstates $\nu_{a}$ and $\nu_{b}$ is washed out.

\footnotetext{
${ }^{1}$ It is necessary to measure accurately the momentum of all the particles because from Eq.(5) we have $\sigma_{p D}^{2}=\sigma_{p D_{I}}^{2}+\sigma_{p D_{F}}^{2}+\sigma_{p \beta}^{2}$.
} 


\section{ACKNOWLEDGMENTS}

C.G. would like to thank W. Grimus for very useful discussions. C.G. and U.W.L. would like to thank the Korea Institute for Advanced Study for their support and warm

hospitality. This work is supported in part by the Ministry of Education through the Basic Science Research Institute, Contract No. BSRI-96-2418(UWL). 


\section{REFERENCES}

[1] S. Nussinov, Phys. Lett. B 63, 201 (1976).

[2] S.M. Bilenky and B. Pontecorvo, Phys. Rep. 41, 225 (1978).

[3] B. Kayser, Phys. Rev. D 24, 110 (1981).

[4] C. Giunti, C.W. Kim and U.W. Lee, Phys. Rev. D 44, 3635 (1991).

[5] C. Giunti, C.W. Kim, J.A. Lee and U.W. Lee, Phys. Rev. D 48, 4310 (1993).

[6] J. Rich, Phys. Rev. D 48, 4318 (1993).

[7] K. Kiers, S. Nussinov and N. Weiss, Phys. Rev. D 53, 537 (1996).

[8] W. Grimus and P. Stockinger, Phys. Rev. D 54, 3414 (1996).

[9] J.E. Campagne, Phys. Lett. B 400, 135 (1997). 